\title{
Isoperimetric Inequality in the Grushin Plane
}

\author{
By Roberto Monti and Daniele Morbidelli
}

\begin{abstract}
We prove a sharp isoperimetric inequality in the Grushin plane and compute the corresponding isoperimetric sets.
\end{abstract}

\section{Introduction}

Carnot-Carathéodory spaces have a metric (Hausdorff) dimension, say $d>2$, larger than their topological dimension, and the isoperimetric inequality gives an upper bound for the volume of bounded sets (their $d$ dimensional Hausdorff measure) in terms of the $d-1$ dimensional Hausdorff measure of the boundary. Apparently, the first result of this type is due to Pansu in the case of the Heisenberg group [19]. In more general Carnot-Carathéodory spaces, isoperimetric inequalities are discussed by Gromov in [12]. These inequalities have also been obtained under the form of Sobolev-Poincaré inequalities. Relationships between isoperimetric inequalities, Sobolev inequalities, and heat kernels are discussed in various settings in [21, 9, 4, 11]. Recently, a geometric measure theory approach to isoperimetric problems in Carnot groups has also been proposed in [14].

In all previous results, the price paid for generality is the lack of sharp constants and extremal sets. In this work we find explicit solutions and sharp constants for the isoperimetric inequality in one of the simplest examples of sub-Riemannian geometry, the "Grushin plane." This expression stems from the hypoelliptic differential operator $\partial_{x}^{2}+x^{2} \partial_{y}^{2}$, which is known as the Grushin operator. We recall the construction of the Grushin plane in a slightly more general situation. Fix a real number $\alpha \geq 0$ and consider the "Riemannian" metric in $\mathbb{R}^{2} d s^{2}=d x^{2}+|x|^{-2 \alpha} d y^{2}$. It is easy to check that, in spite of the singular term, for any pair of points $p, q \in \mathbb{R}^{2}$, there are paths of finite length in the metric $d s^{2}$ connecting $p$ and $q$. Then a distance $d_{\alpha}(p, q)$ can be naturally defined minimizing the length of such curves. The size of the corresponding balls $B_{\alpha}(p, r)$ has been described by Franchi and Lanconelli [10] by means of the boxes

$$
Q_{\alpha}((x, y), r)=[x-r, x+r] \times\left[y-r(|x|+r)^{\alpha}, y+r(|x|+r)^{\alpha}\right] .
$$

Precisely, there exist constants $0<c_{1}<c_{2}$ such that $Q_{\alpha}\left(p, c_{1} r\right) \subset B_{\alpha}(p, r) \subset Q_{\alpha}\left(p, c_{2} r\right)$ for all $p \in \mathbb{R}^{2}$ and $r>0$. The size of small $\alpha$-balls with center away from $x=0$ is approximately of

Math Subject Classifications. 53C17.

Key Words and Phrases. Isoperimetric inequality, Grushin plane. 
Euclidean type. But the Lebesgue measure of $B_{\alpha}((0, y), r)$ is comparable to $r^{2+\alpha}$. The number $d=2+\alpha$ turns out to be the isoperimetric dimension of the Grushin plane.

The $\alpha$-perimeter of a measurable set $E \subset \mathbb{R}^{2}$ can be defined as follows:

$$
P_{\alpha}(E)=\sup \left\{\int_{E}\left(\partial_{x} \varphi_{1}+|x|^{\alpha} \partial_{y} \varphi_{2}\right) d x d y: \varphi_{1}, \varphi_{2} \in C_{0}^{1}\left(\mathbb{R}^{2}\right), \sup _{\mathbb{R}^{2}}\left(\varphi_{1}^{2}+\varphi_{2}^{2}\right)^{1 / 2} \leq 1\right\}
$$

This definition is a special case of the one given in [11] for Carnot-Carathéodory spaces and also of the one introduced in [1] for more general metric spaces. An essential feature of $P_{\alpha}$ is the lower semicontinuity with respect to the $\mathrm{L}^{1}$ convergence of sets, which, along with a compactness argument, easily gives the existence of isoperimetric sets.

The relation between $P_{\alpha}$ and the Grushin metric $d_{\alpha}$ can be described in terms of Minkowski content. Precisely, if $E$ is a bounded open set with regular boundary, then

$$
P_{\alpha}(E)=\mathfrak{M}_{\alpha}(\partial E):=\lim _{\varepsilon \downarrow 0} \frac{\left|\left\{p \in \mathbb{R}^{2}: 0<\operatorname{dist}_{\alpha}(p ; E)<\varepsilon\right\}\right|}{\varepsilon},
$$

where $|\cdot|$ stands for Lebesgue measure in the plane. This identity holds in general CarnotCarathéodory spaces (see [17]). According to a general result of Ambrosio [1], a representation for $\alpha$-perimeter in terms of Hausdorff measures is also possible. Note that, even for a smooth set $E, P_{\alpha}(E)$ is different from the length of $\partial E$ as curve in $\left(\mathbb{R}^{2}, d_{\alpha}\right)$.

We are ready for the statement of our main result.

Theorem 1.1. Let $\alpha \geq 0$ and $d=\alpha+2$. Then for any measurable set $E \subset \mathbb{R}^{2}$ with finite measure

$$
|E| \leq \frac{\alpha+1}{\alpha+2}\left(2 \int_{0}^{\pi} \sin ^{\alpha}(t) d t\right)^{-\frac{1}{\alpha+1}} P_{\alpha}(E)^{\frac{d}{d-1}} .
$$

Equality holds in (1.2) for the isoperimetric set

$$
E_{\alpha}=\left\{(x, y) \in \mathbb{R}^{2}:|y|<\int_{\arcsin |x|}^{\pi / 2} \sin ^{\alpha+1}(t) d t,|x|<1\right\} .
$$

If $\alpha>0$, isoperimetric sets are unique up to vertical translations and dilations of the form $(x, y) \mapsto \delta_{\lambda}(x, y)=\left(\lambda x, \lambda^{\alpha+1} y\right), \lambda>0$.

We briefly explain the proof. The perimeter $P_{\alpha}$ is $(d-1)$-homogeneous with respect to the dilations $\delta_{\lambda}$, whereas Lebesgue measure is $d$-homogeneous (see Proposition 2.2). Using these homogeneity properties, the problem of finding the sharp constant in (1.2) can be reduced to solving the minimum problem

$$
\min \left\{P_{\alpha}(E): E \subset \mathbb{R}^{2} \text { measurable set with }|E|=1\right\} .
$$

A key step in the proof of existence of solutions is to show that the class of admissible sets can be restricted to sets which are symmetric both in the $x$ and in the $y$ direction. In fact, solutions must be symmetric with respect to the $y$ axis. The argument relies upon an adaptation of Steiner symmetrization. After a suitable change of variable $\Psi$, the $\alpha$-perimeter of a set $E$ is equal to the Euclidean perimeter of the set $F=\Psi(E)$ (see Proposition 2.3). By a fundamental result of De Giorgi [6], the Euclidean perimeter of the Steiner symmetrized set $F^{*}$ is less or equal than that of 
$F$. It follows that $P_{\alpha}\left(\Psi^{-1}\left(F^{*}\right)\right) \leq P_{\alpha}(E)$ and the problem is reduced to studying how the map $\Psi$ changes volume (see Theorem 3.1 ).

Our approach is inspired by De Giorgi's original work on the isoperimetric property of balls. Unfortunately, this work is mentioned neither in the rich historical survey of Osserman [18] nor in the comprehensive book of Chavel [5].

Besides symmetry, solutions to problem (1.4) must also be convex. This implies Lipschitz regularity of the boundary of minimum sets, and then, using an integral representation for $\alpha$ perimeter proved in Theorem 2.1, it is possible to write down the Euler-Lagrange equation for problem (1.4), a simple ordinary differential equation that yields the explicit solutions (1.3).

By an argument of Federer and Fleming [8] and Maz'ya [15], inequality (1.2) yields a Gagliardo-Nirenberg inequality for the Grushin sub-elliptic gradient. Precisely,

$$
\left(\int_{\mathbb{R}^{2}}|f|^{\frac{d}{d-1}} d x d y\right)^{\frac{d-1}{d}} \leq c(\alpha)^{\frac{d-1}{d}} \int_{\mathbb{R}^{2}}\left[\left|\partial_{x} f\right|^{2}+|x|^{2 \alpha}\left|\partial_{y} f\right|^{2}\right]^{1 / 2} d x d y
$$

for all functions $f \in C_{0}^{\infty}\left(\mathbb{R}^{2}\right)$. Here $c(\alpha):=\frac{\alpha+1}{\alpha+2}\left(2 \int_{0}^{\pi} \sin ^{\alpha}(t) d t\right)^{-\frac{1}{\alpha+1}}$ is the same constant appearing in the right-hand side of (1.2) and the inequality is sharp (Corollary 4.2). However, contrary to the Euclidean setting, the isoperimetric inequality does not provide the sharp constant in the Sobolev embedding

$$
\left(\int_{\mathbb{R}^{2}}|f|^{\frac{2 d}{d-2}} d x d y\right)^{\frac{d-2}{d}} \leq c \int_{\mathbb{R}^{2}}\left[\left|\partial_{x} f\right|^{2}+|x|^{2 \alpha}\left|\partial_{y} f\right|^{2}\right] d x d y .
$$

Indeed, extremal functions for inequality (1.5) in the case $\alpha=1$ have been recently found by Beckner in [2]. They are functions of the form $f(x, y)=\left(\left(1+x^{2}\right)^{2}+4 y^{2}\right)^{-1 / 4}$, and their level sets are not isoperimetric balls.

Taking (1.1) into account, a simple corollary of (1.2) is the inequality $|E| \leq c(\alpha) \mathfrak{M}_{\alpha}(\partial E)^{\frac{d}{d-1}}$ for bounded open sets (Corollary 4.1). This is the kind of isoperimetric inequality suggested by Gromov in [12] for non equiregular sub-Riemannian manifolds.

Finally, we point out the special interest of the case $\alpha=1$ in connection with the Heisenberg group. In this particular case the isoperimetric ball

$$
E_{1}=\left\{(x, y) \in \mathbb{R}^{2}:|y|<\frac{1}{2}\left(\arccos |x|+|x| \sqrt{1-|x|^{2}}\right),|x|<1\right\}
$$

is bounded by two geodesics for the Grushin metric $d_{1}$ which are symmetric with respect to the $y$ axis (see end of Section 4). The same phenomenon seems to appear in the Heisenberg group, as conjectured by Pansu in [19]. Moreover, identifying the Grushin plane with a vertical hyperplane of $\mathbb{R}^{3}$ and the $y$ axis with the vertical axis of $\mathbb{R}^{3}$, then by rotating $E_{1}$ around the vertical axis one obtains a set which is believed to solve the isoperimetric problem in the Heiseneberg group (see also $[16,13,7])$.

\section{Preliminary results}

In this section we prove some preliminary results concerning $\alpha$-perimeter. Introduce the family of test functions

$$
\mathcal{F}\left(\mathbb{R}^{2}\right)=\left\{\varphi=\left(\varphi_{1}, \varphi_{2}\right) \in C_{0}^{1}\left(\mathbb{R}^{2} ; \mathbb{R}^{2}\right):\|\varphi\|_{\infty} \leq 1\right\},
$$


where $\|\varphi\|_{\infty}=\sup _{\mathbb{R}^{2}}\left(\varphi_{1}^{2}+\varphi_{2}^{2}\right)^{1 / 2}$

We fix a real number $\alpha \geq 0$. The $\alpha$-divergence of a vector valued function $\varphi \in C^{1}\left(\mathbb{R}^{2} ; \mathbb{R}^{2}\right)$ is $\operatorname{div}_{\alpha} \varphi=\partial_{x} \varphi_{1}+|x|^{\alpha} \partial_{y} \varphi_{2}$. Following [11], we define the $\alpha$-perimeter of a measurable subset $E$ of $\mathbb{R}^{2}$ as

$$
P_{\alpha}(E)=\sup _{\varphi \in \mathcal{F}\left(\mathbb{R}^{2}\right)} \int_{E} \operatorname{div}_{\alpha} \varphi(x, y) d x d y .
$$

Two measurable sets $E, F \subset \mathbb{R}^{2}$ are said to be equivalent if $|E \backslash F|=|F \backslash E|=0$. Equivalent sets have the same $\alpha$-perimeter. Our results are stated and hold up to equivalence of sets. If $P_{\alpha}(E)<+\infty$, the set $E$ is said to have finite $\alpha$-perimeter. We shall only consider sets $E$ with finite measure $|E|<+\infty$. In the sequel, when $\alpha=0$ we shall omit the subscript $\alpha$, reducing our definitions to the classical (Euclidean) ones.

A key feature of definition (2.1) is the following lower semicontinuity property. Let $\left(E_{h}\right)_{h \in \mathbb{N}}$ be a sequence of measurable sets whose characteristic functions are converging in $L_{\text {loc }}^{1}\left(\mathbb{R}^{2}\right)$ to the characteristic function of a set $E$. Then

$$
P_{\alpha}(E) \leq \liminf _{h \rightarrow \infty} P_{\alpha}\left(E_{h}\right)
$$

Such a lower semicontinuity and a compactness argument will give the existence of isoperimetric sets.

When the set $E$ has regular boundary, its $\alpha$-perimeter has the following integral representation.

Theorem 2.1. Let $E \subset \mathbb{R}^{2}$ be a bounded open set with Lipschitz boundary. Then

$$
P_{\alpha}(E)=\int_{\partial E}\left(n_{1}(x, y)^{2}+|x|^{2 \alpha} n_{2}(x, y)^{2}\right)^{1 / 2} d \mathcal{H}^{1},
$$

where $n(x, y)=\left(n_{1}(x, y), n_{2}(x, y)\right)$ is the (outward) unit normal to $\partial E$ at the point $(x, y) \in \partial E$, and $\mathcal{H}^{1}$ is the one-dimensional Hausdorff measure in the plane.

Proof. Since $\partial E$ is locally the graph of Lipschitz functions, the normal $n(x, y)$ is defined for $\mathcal{H}^{1}$-a.e. $(x, y) \in \partial E$ and is a $\mathcal{H}^{1}$-measurable function on $\partial E$. Let $F \subset \partial E$ be the set of points of $\partial E$ where $n$ is defined.

Fix a test function $\varphi \in \mathcal{F}\left(\mathbb{R}^{2}\right)$ and recall that $\|\varphi\|_{\infty} \leq 1$. Using the divergence theorem and the Cauchy-Schwarz inequality we get

$$
\int_{E} \operatorname{div}_{\alpha} \varphi d x d y=\int_{\partial E}\left(n_{1} \varphi_{1}+|x|^{\alpha} n_{2} \varphi_{2}\right) d \mathcal{H}^{1} \leq \int_{\partial E}\left(n_{1}^{2}+|x|^{2 \alpha} n_{2}^{2}\right)^{1 / 2} d \mathcal{H}^{1}:=I .
$$

The inequality $P_{\alpha}(E) \leq I$ follows by taking the supremum over all test functions.

We have to prove the converse inequality $I \leq P_{\alpha}(E)$. Note first that the set $G=\{(x, y) \in$ $F: x=0$ and $\left.n_{1}(x, y)=0\right\}$ is at most countable, because it is discrete. Fix a number $\varepsilon>0$. By Lusin theorem there exists a compact set $K \subset F \backslash G$ such that $n_{\mid K}$ is continuous on $K$ and $\mathcal{H}^{1}(\partial E \backslash K) \leq \varepsilon$. Let $B=\left\{(x, y) \in \mathbb{R}^{2}: x^{2}+y^{2} \leq 1\right\}, Q=[-1,1] \times[-1,1]$. Fix a homeomorphism $g: B \rightarrow Q$.

The function $v: K \rightarrow B$ defined by

$$
\nu(x, y)=\frac{\left(n_{1}(x, y),|x|^{\alpha} n_{2}(x, y)\right)}{\left[n_{1}(x, y)^{2}+|x|^{2 \alpha} n_{2}(x, y)^{2}\right]^{1 / 2}}, \quad(x, y) \in K,
$$


is continuous on $K$. The map $g \circ v: K \rightarrow Q$ can be extended to a continuous function from $\mathbb{R}^{2}$ to $Q$ with compact support (this can be seen by applying Tietze-Urysohn theorem to both its components). Taking the composition of this function with $g^{-1}$ we find a continuous function $\psi \in C_{0}\left(\mathbb{R}^{2} ; B\right)$ such that $\psi=v$ on $K$. Write

$$
I=\int_{\partial E}\left(n_{1} \psi_{1}+|x|^{\alpha} n_{2} \psi_{2}\right) d \mathcal{H}^{1}-\int_{\partial E \backslash K}\left(n_{1} \psi_{1}+|x|^{\alpha} n_{2} \psi_{2}-\left(n_{1}^{2}+|x|^{2 \alpha} n_{2}^{2}\right)^{1 / 2}\right) d \mathcal{H}^{1}
$$

Since $\mathcal{H}^{1}(\partial E \backslash K) \leq \varepsilon,\|n\|_{\infty} \leq 1$ and $\|\psi\|_{\infty} \leq 1$, there exists a constant $C$ depending on $\alpha$ and $E$ such that

$$
\left.\int_{\partial E \backslash K}\left|n_{1} \psi_{1}+\right| x\right|^{\alpha} n_{2} \psi_{2}-\left(n_{1}^{2}+|x|^{2 \alpha} n_{2}^{2}\right)^{1 / 2} \mid d \mathcal{H}^{1} \leq C \varepsilon
$$

Then it follows that

$$
\int_{\partial E}\left(n_{1} \psi_{1}+|x|^{\alpha} n_{2} \psi_{2}\right) d \mathcal{H}^{1} \geq I-C \varepsilon
$$

Let $\left(J_{\eta}\right)_{\eta>0}$ be a family of mollifiers and define $\psi_{\eta}=J_{\eta} * \psi$. Then $\psi_{\eta} \in C_{0}^{\infty}\left(\mathbb{R}^{2} ; \mathbb{R}^{2}\right)$, $\left\|\psi_{\eta}\right\|_{\infty} \leq 1$ and $\psi_{\eta} \rightarrow \psi$ uniformly as $\eta \rightarrow 0$. Choosing $\varphi=\psi_{\eta}$ with $\eta>0$ small enough we get

$$
\int_{E} \operatorname{div}_{\alpha} \varphi d x d y=\int_{\partial E}\left(n_{1} \varphi_{1}+|x|^{\alpha} n_{2} \varphi_{2}\right) d \mathcal{H}^{1} \geq I-2 C \varepsilon
$$

and since $\varphi \in \mathcal{F}\left(\mathbb{R}^{2}\right)$ we have $P_{\alpha}(E) \geq I-2 C \varepsilon$. But $\varepsilon>0$ is arbitrary. Then the claim $P_{\alpha}(E) \geq I$ is proved.

Consider the real number $d=2+\alpha$. Lebesgue measure and $\alpha$-perimeter are, respectively, $d$-homogeneous and $(d-1)$-homogeneous with respect to the dilations $(x, y) \mapsto \delta_{\lambda}(x, y)=$ $\left(\lambda x, \lambda^{\alpha+1} y\right)$.

Proposition 2.2. Let $E \subset \mathbb{R}^{2}$ be a measurable set. Then for all $\lambda>0$

(i) $\left|\delta_{\lambda}(E)\right|=\lambda^{d}|E|$;

(ii) $P_{\alpha}\left(\delta_{\lambda}(E)\right)=\lambda^{d-1} P_{\alpha}(E)$.

Proof. We prove (ii). Let $\varphi \in \mathcal{F}\left(\mathbb{R}^{2}\right)$ and write

$$
\begin{aligned}
\int_{\delta_{\lambda}(E)} \operatorname{div}_{\alpha} \varphi(x, y) d x d y & =\int_{\delta_{\lambda}(E)}\left(\partial_{x} \varphi_{1}(x, y)+|x|^{\alpha} \partial_{y} \varphi_{2}(x, y)\right) d x d y \\
& =\int_{E}\left(\frac{1}{\lambda} \partial_{\xi} \varphi_{1}\left(\lambda \xi, \lambda^{\alpha+1} \eta\right)+\lambda^{\alpha}|\xi|^{\alpha} \frac{1}{\lambda^{\alpha+1}} \partial_{\eta} \varphi_{2}\left(\lambda \xi, \lambda^{\alpha+1} \eta\right)\right) \lambda^{d} d \xi d \eta \\
& =\lambda^{d-1} \int_{E} \operatorname{div}_{\alpha}\left(\varphi \circ \delta_{\lambda}\right)(\xi, \eta) d \xi d \eta \leq \lambda^{d-1} P_{\alpha}(E),
\end{aligned}
$$

because $\varphi \circ \delta_{\lambda} \in \mathcal{F}\left(\mathbb{R}^{2}\right)$. Taking the supremum over test functions gives $P_{\alpha}\left(\delta_{\lambda}(E)\right) \leq \lambda^{d-1} P_{\alpha}$ $(E)$. The converse inequality is obtained in the same way.

We introduce a change of variable that transforms the $\alpha$-perimeter of a set into the usual perimeter of its transformed set. Consider the functions $\Phi, \Psi: \mathbb{R}^{2} \rightarrow \mathbb{R}^{2}$ defined by

$$
\Phi(\xi, \eta)=\left(\operatorname{sgn}(\xi)|(\alpha+1) \xi|^{\frac{1}{\alpha+1}}, \eta\right), \quad \Psi(x, y)=\left(\operatorname{sgn}(x) \frac{|x|^{\alpha+1}}{\alpha+1}, y\right)
$$


Clearly, $\Psi$ is a homeomorphism and $\Phi$ is its inverse. Notice that $|\operatorname{det} J \Phi(\xi, \eta)|=|(\alpha+1) \xi|^{-\frac{\alpha}{\alpha+1}}$ for $\xi \neq 0$.

Proposition 2.3. Let $E \subset \mathbb{R}^{2}$ be a measurable set and define $F=\Psi(E)$. Then $P(F)=$ $P_{\alpha}(E)$.

Proof. Take a test function $\varphi \in \mathcal{F}\left(\mathbb{R}^{2}\right)$. A short computation gives

$$
\begin{aligned}
\int_{E} \operatorname{div}_{\alpha} \varphi(x, y) d x d y & =\int_{E}\left[\partial_{x} \varphi_{1}(x, y)+|x|^{\alpha} \partial_{y} \varphi_{2}(x, y)\right] d x d y \\
& =\int_{F}\left[\partial_{\xi}\left(\varphi_{1} \circ \Phi\right)(\xi, \eta)+\partial_{\eta}\left(\varphi_{2} \circ \Phi\right)(\xi, \eta)\right] d \xi d \eta
\end{aligned}
$$

Note that the function $\partial_{\xi}\left(\varphi_{1} \circ \Phi\right)(\xi, \eta)=|(\alpha+1) \xi|^{-\frac{\alpha}{\alpha+1}}\left(\partial_{1} \varphi_{1}\right)(\Phi(\xi, \eta))$ is in $L^{1}\left(\mathbb{R}^{2}\right)$, because $\partial_{1} \varphi_{1}$ is bounded and with compact support, and the singular term $|\xi|^{-\frac{\alpha}{\alpha+1}}$ is locally integrable. The same happens for $\partial_{\eta}\left(\varphi_{2} \circ \Phi\right)$.

By known density theorems for Sobolev spaces

$$
\begin{aligned}
P(F) & =\sup _{\psi \in \mathcal{F}\left(\mathbb{R}^{2}\right)} \int_{F} \operatorname{div} \psi(\xi, \eta) d \xi d \eta \\
& =\sup \left\{\int_{F} \operatorname{div} \psi(\xi, \eta) d \xi d \eta: \psi_{1}, \psi_{2} \in \mathrm{W}^{1,1}\left(\mathbb{R}^{2}\right), \psi_{1}^{2}+\psi_{2}^{2} \leq 1 \text { a.e. }\right\} .
\end{aligned}
$$

Then it follows that

$$
\int_{E} \operatorname{div}_{\alpha} \varphi(x, y) d x d y \leq P(F)
$$

Taking the supremum over test functions we find $P_{\alpha}(E) \leq P(F)$. The converse inequality can be achieved by the same argument, using the function $\Psi$ instead of $\Phi$.

\section{Isoperimetric inequality}

In this section we prove the isoperimetric inequality. First we need a theorem that reduces the problem to convex and symmetric sets. To this aim we introduce some definitions concerning geometrical properties of sets. A set $E \subset \mathbb{R}^{2}$ is $x$-symmetric if $(x, y) \in E$ implies $(-x, y) \in E$. $E$ is $y$-symmetric if $(x, y) \in E$ implies $(x,-y) \in E$. Finally, $E$ is said to be symmetric if it is both $x$-and $y$-symmetric.

Given a set $E \subset \mathbb{R}^{2}$ define for every $x, y \in \mathbb{R}$

$$
E^{x}=\{y \in \mathbb{R}:(x, y) \in E\}, \quad E^{y}=\{x \in \mathbb{R}:(x, y) \in E\} .
$$

A set $E \subset \mathbb{R}^{2}$ is $x$-convex if $E^{y}$ is an (open or empty) interval for all $y \in \mathbb{R}$. $E$ is $y$-convex if $E^{x}$ is an (open or empty) interval for all $x \in \mathbb{R}$. Finally, $E$ will be said to be separately convex if it is both $x$ - and $y$-convex.

Theorem 3.1. Let $E \subset \mathbb{R}^{2}$ be a measurable set with $P_{\alpha}(E)<+\infty$ and $0<|E|<+\infty$. There exists a symmetric, convex set $E^{*} \subset \mathbb{R}^{2}$ such that $P_{\alpha}\left(E^{*}\right) \leq P_{\alpha}(E)$ and $\left|E^{*}\right|=|E|$. Moreover, in case $\alpha>0$, if $E$ is not (equivalent to) an $x$-symmetric and convex set, then the strict inequality $P_{\alpha}\left(E^{*}\right)<P_{\alpha}(E)$ holds. 
Proof. Let $E \subset \mathbb{R}^{2}$ be a measurable set with positive and finite measure and finite perimeter. Define $F=\Psi(E)$, where $\Psi$ is the map introduced in (2.4). By Proposition 2.3, $P(F)=P_{\alpha}(E)<$ $+\infty$. Moreover, letting

$$
\mu(F)=\int_{F}|(\alpha+1) \xi|^{\beta} d \xi d \eta, \quad \beta=-\frac{\alpha}{\alpha+1},
$$

we find

$$
|E|=\int_{\Phi(F)} d x d y=\int_{F}|\operatorname{det} J \Phi(\xi, \eta)| d \xi d \eta=\mu(F) .
$$

Let $F_{1}$ be the Steiner symmetrization of $F$ in the $\eta$-direction. Precisely,

$$
F_{1}=\left\{(\xi, \eta) \in \mathbb{R}^{2}:|\eta|<\frac{1}{2}\left|F^{\xi}\right|\right\} .
$$

Here, $|\cdot|$ stands for one-dimensional Lebesgue measure. By [6, Theorem II], (see also [20, Section 3.8]), $P\left(F_{1}\right) \leq P(F)$, where the inequality is strict if $F$ is not (equivalent to) an $\eta$ convex set. Moreover, by the Fubini-Tonelli Theorem

$$
\begin{aligned}
\mu(F) & =\int_{F}|(\alpha+1) \xi|^{\beta} d \xi d \eta=\int_{-\infty}^{+\infty}|(\alpha+1) \xi|^{\beta}\left|F^{\xi}\right| d \xi \\
& =\int_{-\infty}^{+\infty}|(\alpha+1) \xi|^{\beta}\left|F_{1}^{\xi}\right| d \xi=\mu\left(F_{1}\right),
\end{aligned}
$$

because $\left|F^{\xi}\right|=\left|F_{1}^{\xi}\right|$ for all $\xi \in \mathbb{R}$.

Let $F_{2}$ be the Steiner symmetrization of $F_{1}$ in the $\xi$-direction. Precisely,

$$
F_{2}=\left\{(\xi, \eta) \in \mathbb{R}^{2}:|\xi|<\frac{1}{2}\left|F_{1}^{\eta}\right|\right\} .
$$

Then, as above, $P\left(F_{2}\right) \leq P\left(F_{1}\right) \leq P(F)$. Consider the volume

$$
\mu\left(F_{2}\right)=\int_{F_{2}}|(\alpha+1) \xi|^{\beta} d \xi d \eta=\int_{-\infty}^{+\infty}\left(\int_{F_{2}^{\eta}}|(\alpha+1) \xi|^{\beta} d \xi\right) d \eta
$$

In order to estimate the last term, we use the following elementary fact. Given a measurable set $I \subset \mathbb{R}$ with finite measure, denote by $\left.I^{*}=(-|I| / 2,|I| / 2)\right)$ its symmetrized set. Since the number $\beta$ is negative, we have $|\xi|^{\beta} \geq(|I| / 2)^{\beta}$ if $\xi \in I^{*}$, and $|\xi|^{\beta} \leq(|I| / 2)^{\beta}$ if $\xi \in I \backslash I^{*}$. Thus,

$$
\begin{aligned}
\int_{I}|\xi|^{\beta} d \xi & =\int_{I \cap I^{*}}|\xi|^{\beta} d \xi+\int_{I \backslash I^{*}}|\xi|^{\beta} d \xi \leq \int_{I \cap I^{*}}|\xi|^{\beta} d \xi+\left(\frac{|I|}{2}\right)^{\beta}\left|I \backslash I^{*}\right| \\
& =\int_{I \cap I^{*}}|\xi|^{\beta} d \xi+\left(\frac{|I|}{2}\right)^{\beta}\left|I^{*} \backslash I\right| \leq \int_{I^{*}}|\xi|^{\beta} d \xi
\end{aligned}
$$

The inequality is strict if and only if $I$ is equivalent to $I^{*}$.

From the above considerations it follows that $\mu\left(F_{2}\right) \geq \mu\left(F_{1}\right)$ with equality if and only if $F_{1}$ is (equivalent to) an $x$-symmetric and $x$-convex set.

$F_{2}$ is a symmetric, separately convex open set. Moreover, $\partial F_{2}$ is the union of the image of four 1-Lipschitz curves. This can be easily visualized by looking at the set after a rotation of 45 
degrees. More precisely, for all $s \in \mathbb{R}$ such that the set written below is nonempty, define the function

$$
\vartheta(s)=\sup \left\{t>|s|:\left(\frac{t+s}{\sqrt{2}}, \frac{t-s}{\sqrt{2}}\right) \in F_{2}\right\} .
$$

$F_{2}$ is separately convex and then $\vartheta$ is 1-Lipschitz. Moreover, $\partial F_{2} \cap\{\xi>0, \eta>0\}$ is a graph of the form $t=\vartheta(s)$ in the variables $s=(\xi-\eta) / \sqrt{2}$ and $t=(\xi+\eta) / \sqrt{2}$. From a well-known characterization of Euclidean perimeter, it follows that $P\left(F_{2}\right)=\mathcal{H}^{1}\left(\partial F_{2}\right)$.

Let $F_{3}=\operatorname{co}\left(F_{2}\right)$ be the convex hull of $F_{2}$. Since $F_{2} \subset F_{3}$, it follows that $\mu\left(F_{2}\right) \leq \mu\left(F_{3}\right)$ with strict inequality if $F_{2}$ is not a convex set. Write $\partial F_{3}=\left(\partial F_{3} \cap \partial F_{2}\right) \cup\left(\partial F_{3} \backslash \partial F_{2}\right)$, where $\partial F_{3} \backslash \partial F_{2}$ is the disjoint union of an at most countable family of line segments $I_{n}=\left(p_{n}, q_{n}\right) \subset \mathbb{R}^{2}$, $n \in \mathbb{N}$. Analogously, $\partial F_{2}=\left(\partial F_{2} \cap \partial F_{3}\right) \cup\left(\partial F_{2} \backslash \partial F_{3}\right)$, where $\partial F_{2} \backslash \partial F_{3}$ is the disjoint union of an at most countable family of rectifiable curves $\gamma_{n}, n \in \mathbb{N}$. After a relabelling, we can assume that $\gamma_{n}$ connects $p_{n}$ and $q_{n}$. Then the length of $\gamma_{n}$ is greater than that of $I_{n}$, and therefore $P\left(F_{3}\right)=\mathcal{H}^{1}\left(\partial F_{3}\right) \leq \mathcal{H}^{1}\left(\partial F_{2}\right)=P\left(F_{2}\right)$.

Define $E^{*}=\delta_{\lambda}\left(\Phi\left(F_{3}\right)\right)$, where $\lambda>0$ is chosen in order to ensure $\left|E^{*}\right|=|E|$ (it turns out that $\lambda \leq 1$, see below). The set $E^{*}$ is symmetric because $\Phi$ preserves symmetry. We show that $E^{*}$ is also convex. Since the map $\delta_{\lambda}$ is linear, it is sufficient to show that $\Phi\left(F_{3}\right)$ is convex. Let $\left(x_{0}, y_{0}\right),\left(x_{1}, x_{1}\right) \in \Phi\left(F_{3}\right)$ and write $\left(x_{i}, y_{i}\right)=\Phi\left(\xi_{i}, \eta_{i}\right),\left(\xi_{i}, \eta_{i}\right) \in F_{3}, i=0,1$. $\Phi\left(F_{3}\right)$ is symmetric and separately convex and therefore we can without loss of generality assume $x_{0}, x_{1} \geq 0$. Clearly, $\Phi\left(\tau\left(\xi_{0}, \eta_{0}\right)+(1-\tau)\left(\xi_{1}, \eta_{1}\right)\right) \in \Phi\left(F_{3}\right), \tau \in[0,1]$, because $F_{3}$ is convex. From the concavity inequality

$$
\tau \xi_{0}^{\frac{1}{\alpha+1}}+(1-\tau) \xi_{1}^{\frac{1}{\alpha+1}} \leq\left(\tau \xi_{0}+(1-\tau) \xi_{1}\right)^{\frac{1}{\alpha+1}}, \quad \tau \in[0,1], \xi_{0}, \xi_{1} \geq 0
$$

and from $x$-symmetry, $x$ - and $y$-convexity of $\Phi\left(F_{3}\right)$, it follows that $\tau \Phi\left(\xi_{0}, \eta_{0}\right)+(1-\tau) \Phi\left(\xi_{1}, \eta_{1}\right)$ $\in \Phi\left(F_{3}\right)$ for all $\tau \in[0,1]$.

Notice that $\left|\Phi\left(F_{3}\right)\right|=\mu\left(F_{3}\right) \geq \mu\left(F_{2}\right) \geq \mu\left(F_{1}\right)=\mu(F)=|E|$, and then it must be $\lambda \leq 1$, with $\lambda<1$ if $E$ is not (equivalent to) an $x$-symmetric, convex set. Moreover, by Propositions 2.2 and 2.3 it follows that

$$
\lambda^{1-d} P_{\alpha}\left(E^{*}\right)=P_{\alpha}\left(\Phi\left(F_{3}\right)\right)=P\left(F_{3}\right) \leq P\left(F_{2}\right) \leq P(F)=P_{\alpha}(E) .
$$

Hence, $P_{\alpha}\left(E^{*}\right) \leq P_{\alpha}(E)$ with strict inequality if $E$ is not (equivalent to) an $x$-symmetric, convex set.

A measurable set with positive and finite measure minimizing the ratio $P_{\alpha}(E)^{\frac{d}{d-1}} /|E|$ will be called an isoperimetric set. The class of isoperimetric sets is invariant under dilations $(x, y) \mapsto$ $\delta_{\lambda}(x, y), \lambda>0$, and under vertical translations $(x, y) \mapsto(x, y+h), h \in \mathbb{R}$.

Theorem 3.2. Let $\alpha>0$ and $d=\alpha+2$. There exists a constant $c(\alpha)>0$ such that for any measurable set $E \subset \mathbb{R}^{2}$ with finite measure

$$
|E| \leq c(\alpha) P_{\alpha}(E)^{\frac{d}{d-1}} .
$$

The constant $c(\alpha)$ is determined by equality in (3.1) achieved by the isoperimetric set

$$
E_{\alpha}=\left\{(x, y) \in \mathbb{R}^{2}:|y|<\int_{\arcsin |x|}^{\pi / 2} \sin ^{\alpha+1}(t) d t,|x|<1\right\}
$$


precisely,

$$
c(\alpha)=\frac{\alpha+1}{\alpha+2}\left(2 \int_{0}^{\pi} \sin ^{\alpha}(t) d t\right)^{-\frac{1}{\alpha+1}} .
$$

Isoperimetric sets are unique up to dilations and vertical translations.

Proof. Consider the following minimum problem

$$
\min \left\{P_{\alpha}(E): E \subset \mathbb{R}^{2} \text { measurable set with }|E|=1\right\} .
$$

We study the existence of solutions by the direct method of the calculus of variations. By Theorem 3.1 the class of admissible sets can be restricted to symmetric and convex sets. Recall that a set is symmetric if it is both $x$ - and $y$-symmetric. Define

$$
\mathcal{A}=\left\{E \subset \mathbb{R}^{2}: E \text { symmetric, convex set with }|E|=1 \text { and } P_{\alpha}(E) \leq k\right\} .
$$

Here $k>0$ is any fixed constant large enough to ensure $\mathcal{A} \neq \emptyset$. Such a constant does exist.

We claim that any set $E \in \mathcal{A}$ is contained in the rectangle $[-a, a] \times[-b, b]$, where $a>0$ and $b>0$ depend only on $k$ and $\alpha$. Fix a number $\varepsilon>0$. Let $\psi_{\varepsilon} \in C^{l}(\mathbb{R})$ be an increasing function such that $\psi_{\varepsilon}(y)=1$ if $y \geq \varepsilon$ and $\psi_{\varepsilon}(y)=-1$ if $y \leq-\varepsilon$. Take a set $E \in \mathcal{A}$ and let $a=\sup \left\{x>0:\left|E^{x}\right|>0\right\}, b=\sup \left\{y>0:\left|E^{y}\right|>0\right\}, a_{\varepsilon}=\sup \left\{x>0:\left|E^{x}\right|>2 \varepsilon\right\}$ and $b_{\varepsilon}=\sup \left\{y>0:\left|E^{y}\right|>2 \varepsilon\right\}$. The numbers $a_{\varepsilon}$ and $b_{\varepsilon}$ are both finite and tend, respectively to $a$ and $b$, as $\varepsilon \rightarrow 0$. Choose the test function $\varphi_{\varepsilon}(x, y)=\left(0, \vartheta(x, y) \psi_{\varepsilon}(y)\right) \in \mathcal{F}\left(\mathbb{R}^{2}\right)$, where $\vartheta \in C_{0}^{1}\left(\mathbb{R}^{2}\right)$ is a function such that $\chi_{E} \leq \vartheta \leq 1$. We have

$$
\begin{aligned}
k & \geq P_{\alpha}(E) \geq \int_{E}|x|^{\alpha} \partial_{y}\left(\vartheta(x, y) \psi_{\varepsilon}(y)\right) d x d y=\int_{E}|x|^{\alpha} \partial_{y} \psi_{\varepsilon}(y) d x d y \\
& =\int_{-a}^{a}|x|^{\alpha} \int_{E^{x}} \partial_{y} \psi_{\varepsilon}(y) d y d x \geq 2 \int_{-a_{\varepsilon}}^{a_{\varepsilon}}|x|^{\alpha} d x=4 \frac{a_{\varepsilon}^{\alpha+1}}{\alpha+1} .
\end{aligned}
$$

Since $a_{\varepsilon} \rightarrow a$ when $\varepsilon \rightarrow 0$, we get $4 a^{\alpha+1} \leq k(\alpha+1)$. A similar argument shows that $4 b \leq k$. The claim is proved.

Let $\left(E_{h}\right)_{h \in \mathbb{N}} \subset \mathcal{A}$ be a minimizing sequence for problem (3.4)

$$
\lim _{h \rightarrow \infty} P_{\alpha}\left(E_{h}\right)=\inf \left\{P_{\alpha}(E): E \in \mathcal{A}\right\} .
$$

The sets $F_{h}=\Psi\left(E_{h}\right)$ are contained in the bounded set $\Psi([-a, a] \times[-b, b])$. Moreover, by Proposition 2.3, $P\left(F_{h}\right)=P_{\alpha}\left(E_{h}\right) \leq k$ for all $h \in \mathbb{N}$. The space of functions with bounded variation $\mathrm{BV}\left(\mathbb{R}^{2}\right)$ is compactly embedded in $\mathrm{L}_{\text {loc }}^{1}\left(\mathbb{R}^{2}\right)$. Therefore, possibly extracting a subsequence, there exists a measurable set $F \subset \Psi([-a, a] \times[-b, b])$ such that $\chi_{F_{h}} \rightarrow \chi_{F}$ in $\mathrm{L}^{1}\left(\mathbb{R}^{2}\right)$. Letting $E=\Phi(F)$, it follows that $\chi_{E_{h}} \rightarrow \chi_{E}$ in $\mathrm{L}^{1}\left(\mathbb{R}^{2}\right)$. The set $E$ is (equivalent to) an $x-$ and $y$-symmetric and convex set. This follows from the fact that $\chi_{E_{h}}$ can be also assumed to converge almost everywhere to $\chi_{E}$. By the lower semicontinuity (2.2)

$$
P_{\alpha}(E) \leq \liminf _{h \rightarrow \infty} P_{\alpha}\left(E_{h}\right)=\inf \left\{P_{\alpha}(E): E \in \mathcal{A}\right\}
$$

Thus, $E$ is a minimum, because $E \in \mathcal{A}$. By Proposition 2.2 this set is also a solution of the problem

$$
\min \left\{\frac{P_{\alpha}(E)^{\frac{d}{d-1}}}{|E|}: E \subset \mathbb{R}^{2} \text { measurable set with } 0<|E|<+\infty\right\}
$$


The set $E$ is convex and therefore its boundary $\partial E$ is locally the graph of Lipschitz functions. In a neighborhood of the point $(0, b) \in \partial E, b>0$, the set $\partial E$ can be written as a Lipschitz graph of the form $y=\varphi(x)$. We are led to the following situation. Let $\delta>0, \varphi \in \operatorname{Lip}(-\delta, \delta)$ and assume that $\{(x, \varphi(x)): x \in(-\delta, \delta)\}=\partial E \cap\left\{(x, y) \in \mathbb{R}^{2}:-\delta<x<\delta, y>0\right\}$. Fix a function $\vartheta \in C_{0}^{1}(-\delta, \delta)$. For $|t|<t_{0}$ let $E_{t}$ be the set obtained from $E$ by replacing $\partial E \cap\left\{(x, y) \in \mathbb{R}^{2}:-\delta<x<\delta, y>0\right\}$ with $\{(x, \varphi(x)+t \vartheta(x)): x \in(-\delta, \delta)\}$. Denote by $\left(n_{1}^{t}, n_{2}^{t}\right)$ the unit normal to $\partial E_{t}$. By Theorem 2.1 and by the length formula

$$
\begin{aligned}
\left.\frac{d}{d t} P_{\alpha}\left(E_{t}\right)\right|_{t=0} & =\left.\frac{d}{d t} \int_{\partial E_{f} \cap\{|x|<\delta, y>0\}}\left[n_{1}^{t}(x, y)^{2}+|x|^{2 \alpha} n_{2}^{t}(x, y)^{2}\right]^{1 / 2} d \mathcal{H}^{1}\right|_{t=0} \\
& =\left.\frac{d}{d t} \int_{-\delta}^{\delta}\left[\left(\varphi^{\prime}(x)+t \vartheta^{\prime}(x)\right)^{2}+|x|^{2 \alpha}\right]^{1 / 2} d x\right|_{t=0} \\
& =\int_{-\delta}^{\delta} \frac{\varphi^{\prime}(x) \vartheta^{\prime}(x)}{\left[\varphi^{\prime}(x)^{2}+|x|^{2 \alpha}\right]^{1 / 2}} d x
\end{aligned}
$$

We can interchange derivative and integral because

$$
\begin{aligned}
\left|\frac{\partial}{\partial t}\left[\left(\varphi^{\prime}(x)+t \vartheta^{\prime}(x)\right)^{2}+|x|^{2 \alpha}\right]^{1 / 2}\right| & =\frac{\left|\left(\varphi^{\prime}(x)+t \vartheta^{\prime}(x)\right) \vartheta^{\prime}(x)\right|}{\left[\left(\varphi^{\prime}(x)+t \vartheta^{\prime}(x)\right)^{2}+|x|^{2 \alpha}\right]^{1 / 2}} \\
& \leq\left|\vartheta^{\prime}(x)\right| \in \mathrm{L}^{1}(-\delta, \delta) .
\end{aligned}
$$

Analogously,

$$
\left.\frac{d}{d t}\left|E_{t}\right|\right|_{t=0}=\left.\frac{d}{d t} \int_{-\delta}^{\delta}(\varphi(x)+t \vartheta(x)) d x\right|_{t=0}=\int_{-\delta}^{\delta} \vartheta(x) d x=-\int_{-\delta}^{\delta} x \vartheta^{\prime}(x) d x .
$$

The set $E$ is a solution of problem (3.6), and hence

$$
\frac{P_{\alpha}(E)^{\frac{d}{d-1}}}{|E|} \leq \frac{P_{\alpha}\left(E_{t}\right)^{\frac{d}{d-1}}}{\left|E_{t}\right|}, \quad|t|<t_{0}
$$

Thus,

$$
\begin{aligned}
0 & =\left.\frac{d}{d t} \frac{P_{\alpha}\left(E_{t}\right)^{\frac{d}{d-1}}}{\left|E_{t}\right|}\right|_{t=0} \\
& =\frac{P_{\alpha}(E)^{\frac{1}{d-1}}}{|E|^{2}}\left(\frac{d}{d-1}|E| \int_{-\delta}^{\delta} \frac{\varphi^{\prime}(x) \vartheta^{\prime}(x)}{\left[\varphi^{\prime}(x)^{2}+|x|^{2 \alpha}\right]^{1 / 2}} d x+P_{\alpha}(E) \int_{-\delta}^{\delta} x \vartheta^{\prime}(x) d x\right) .
\end{aligned}
$$

The function $\vartheta \in C_{0}^{l}(-\delta, \delta)$ is arbitrary. Therefore it must be

$$
\frac{d}{d-1}|E| \frac{\varphi^{\prime}(x)}{\left[\varphi^{\prime}(x)^{2}+|x|^{2 \alpha}\right]^{1 / 2}}+P_{\alpha}(E) x=c, \quad \text { for a.e. } x \in(-\delta, \delta),
$$

for some constant $c \in \mathbb{R}$. The function $\varphi$ must be even because the set $E$ is $x$-symmetric. Then $\varphi^{\prime}$ is odd and this implies $c=0$. Setting $\lambda=\frac{d-1}{d} \frac{P_{\alpha}(E)}{|E|}$ we find

$$
\varphi^{\prime}(x)=-\operatorname{sgn}(x) \frac{\lambda|x|^{\alpha+1}}{\left[1-\lambda^{2} x^{2}\right]^{1 / 2}} \quad \text { for a.e. } x \in(-\delta, \delta) .
$$


This equation shows that $\varphi^{\prime}$, which a priori is only a locally bounded measurable function, is in fact, a continuous function, and the equation is satisfied for all $|x|<1 / \lambda$.

Letting $a=\sup \left\{x>0:\left|E^{x}\right|>0\right\}$, a regularity argument similar to the one discussed above shows that $\partial E$ is of class $C^{1}$ in a neighborhood of $(a, 0)$. Then it must be $\varphi(a)=0, \varphi^{\prime}(a)=-\infty$ and $a=1 / \lambda$. Hence, for $x \in[0, a]$

$$
\varphi(x)=\int_{x}^{a} \frac{t^{\alpha+1}}{a\left(1-(t / a)^{2}\right)^{1 / 2}} d t=a^{\alpha+1} \int_{\arcsin (x / a)}^{\pi / 2} \sin ^{\alpha+1}(t) d t .
$$

The parameter $a>0$ is fixed by means of the volume constraint $|E|=1$.

If we choose $\lambda=a=1$ then we find the isoperimetric set $E_{\alpha}$ in (3.2). By (3.9) with $\lambda=1$ and Theorem 2.1 we also find

$$
P_{\alpha}\left(E_{\alpha}\right)=4 \int_{0}^{1}\left[\varphi^{\prime}(x)^{2}+|x|^{2 \alpha}\right]^{1 / 2} d x=4 \int_{0}^{1} \frac{|x|^{\alpha}}{\sqrt{1-x^{2}}} d x=2 \int_{0}^{\pi} \sin ^{\alpha}(t) d t
$$

Moreover, $\left|E_{\alpha}\right|=\frac{d-1}{d} P_{\alpha}\left(E_{\alpha}\right)$. Therefore, the isoperimetric constant $c(\alpha)$ is given by

$$
c(\alpha)=\frac{\left|E_{\alpha}\right|}{P_{\alpha}\left(E_{\alpha}\right)^{\frac{d}{d-1}}}=\frac{d-1}{d} P_{\alpha}\left(E_{\alpha}\right)^{\frac{1}{1-d}}=\frac{d-1}{d}\left(2 \int_{0}^{\pi} \sin ^{\alpha}(t) d t\right)^{\frac{1}{1-d}} .
$$

The statement concerning uniqueness follows from Theorem 3.1 and from the previous analysis.

\section{Corollaries and remarks}

In the first part of this section we state some corollaries of Theorem 3.2. In the second one, we discuss the isoperimetric inequality in the Grushin plane in connection with the isoperimetric problem in the Heisenberg group. For technical reasons connected with the Lipschitz continuity of the function $x \mapsto|x|^{\alpha}$, we shall state the next corollaries for the case $\alpha \geq 1$.

Consider a bounded open set $E \subset \mathbb{R}^{2}$ and define the distance $\operatorname{dist}_{\alpha}(p ; E)=\inf _{q \in E} d_{\alpha}(p, q)$. We define the Minkowski content of $\partial E$ as

$$
\mathfrak{M}_{\alpha}(\partial E)=\liminf _{\varepsilon \downarrow 0} \frac{\left|\left\{p \in \mathbb{R}^{2}: 0<\operatorname{dist}_{\alpha}(p ; E)<\varepsilon\right\}\right|}{\varepsilon} .
$$

If $E$ is a bounded open set with boundary of class $C^{2}$, then "lim inf" in (4.1) can be replaced by "lim" and the identity $\mathfrak{M}_{\alpha}(\partial E)=P_{\alpha}(E)$ holds. This can be proved as in [17] Theorem 5.1.

The following corollary gives a sharp isoperimetric inequality for Minkowski content.

Corollary 4.1. Let $\alpha \geq 1, d=2+\alpha$ and let $c(\alpha)$ be the constant in (3.3). Then, for any bounded open set $E \subset \mathbb{R}^{2}$ it holds

$$
|E| \leq c(\alpha) \mathfrak{M}_{\alpha}(\partial E)^{\frac{d}{d-1}}
$$

Proof. Let $E \subset \mathbb{R}^{2}$ be a bounded open set and write $\varrho(p)=\operatorname{dist}_{\alpha}(p ; E)$. For any $\varepsilon>0$ let $E_{\varepsilon}=\left\{p \in \mathbb{R}^{2}: \varrho(p)<\varepsilon\right\}$. Without loss of generality we can assume that $\left|E_{\varepsilon} \backslash E\right|$ converges to zero as $\varepsilon \downarrow 0$, otherwise $\mathfrak{M}_{\alpha}(\partial E)=+\infty$. 
By Theorem 3.1 in [17] we have the Eikonal equation $\left.\left.\left(\mid \partial_{x} \varrho(x, y)\right)\right|^{2}+|x|^{2 \alpha}\left|\partial_{y} \varrho(x, y)\right|^{2}\right)^{1 / 2}$ $=1$ for almost every $(x, y) \in \mathbb{R}^{2} \backslash E$. From the coarea formula proved in Theorem 5.2 of [11] it follows

$$
\left.\left|E_{\varepsilon} \backslash E\right|=\left.\int_{E_{\varepsilon} \backslash E}\left(\mid \partial_{x} \varrho(x, y)\right)\right|^{2}+|x|^{2 \alpha}\left|\partial_{y} \varrho(x, y)\right|^{2}\right)^{1 / 2} d x d y=\int_{0}^{\varepsilon} P_{\alpha}\left(E_{\tau}\right) d \tau .
$$

Given $\varepsilon>0$, it cannot be $P_{\alpha}\left(E_{\tau}\right)>\frac{\left|E_{\varepsilon} \backslash E\right|}{\varepsilon}$ for all $\tau \in(0, \varepsilon)$, otherwise (4.3) would be false. Then, for every $\varepsilon>0$ there exists $\tau(\varepsilon) \in(0, \varepsilon)$ such that

$$
P_{\alpha}\left(E_{\tau(\varepsilon)}\right) \leq \frac{\left|E_{\varepsilon} \backslash E\right|}{\varepsilon} .
$$

From (2.2), by taking the lim inf we find $P_{\alpha}(E) \leq \mathfrak{M}_{\alpha}(\partial E)$ and the claim follows from (3.1).

The second corollary we discuss is a Sobolev inequality for the Grushin gradient.

Corollary 4.2. Let $\alpha \geq 1, d=2+\alpha$ and let $c(\alpha)$ be the constant in (3.3). Then for any $f \in C_{0}^{\infty}\left(\mathbb{R}^{2}\right)$

$$
\left.\left(\int_{\mathbb{R}^{2}}|f|^{\frac{d}{d-1}} d x d y\right)^{\frac{d-1}{d}} \leq\left. c(\alpha)^{\frac{d-1}{d}} \int_{\mathbb{R}^{2}}\left[\mid \partial_{x} f\right)\right|^{2}+|x|^{2 \alpha}\left|\partial_{y} f\right|^{2}\right]^{1 / 2} d x d y
$$

The constant in this inequality is sharp.

Proof. The isoperimetric inequality (3.1) implies (4.4). This can be proved by a straightforward adaptation of the argument in Remark 6.6 of [8].

The sharpness of the constant can by proved in the following way. We take a bounded open set $E \subset \mathbb{R}^{2}$ with boundary of class $C^{2}$ and we define, as before, $\varrho(p)=\operatorname{dist}_{\alpha}(p ; E)$. For any $\varepsilon>0$ let

$$
f_{\varepsilon}(p)= \begin{cases}1 & \text { if } p \in \bar{E} \\ 1-\frac{1}{\varepsilon} \varrho(p) & \text { if } 0<\varrho(p)<\varepsilon \\ 0 & \text { if } \varrho(p) \geq \varepsilon\end{cases}
$$

Apply the Sobolev inequality to $f_{\varepsilon}$. Letting $\varepsilon \rightarrow 0$ and using the Eikonal equation and the identity $\mathfrak{M}_{\alpha}(\partial E)=P_{\alpha}(E)$ we get the isoperimetric inequality (3.1).

Finally, we would like to point out the special interest of the isoperimetric problem in the Grushin plane in the case $\alpha=1$. Geodesics (i.e., curves with minimal length connecting points) in the metric space $\left(\mathbb{R}^{2}, d_{\alpha}\right)$ are solution of a particular system of differential equations. Consider the Hamilton function $H(x, y, \xi, \eta)=\frac{1}{2}\left(\xi^{2}+|x|^{2 \alpha} \eta^{2}\right)$ and the corresponding problem (we study the case $x \geq 0$ )

$$
\begin{cases}\dot{x}=\partial_{\xi} H(x, y, \xi, \eta)=\xi & x(0)=0 \\ \dot{y}=\partial_{\eta} H(x, y, \xi, \eta)=x^{2 \alpha} \eta & y(0)=0 \\ \dot{\xi}=-\partial_{x} H(x, y, \xi, \eta)=-\alpha x^{2 \alpha-1} \eta^{2} & \xi(0)=1 \\ \dot{\eta}=-\partial_{y} H(x, y, \xi, \eta)=0 & \eta(0)=-\lambda\end{cases}
$$

Geodesics have to be found (after a reparameterization) among curves $\gamma(t)=(x(t), y(t))$ solving this problem. We refer to [3] for a motivation of this fact. The choice $x(0)=0$ and $y(0)=0$ 
means that $\gamma$ starts from the origin, whereas the choice $\xi(0)=1$ corresponds to arclength parameterization. Finally, $\lambda>0$ is a parameter controlling the direction of the curve. The first, third, and fourth equations give $\ddot{x}+\alpha \lambda^{2} x^{2 \alpha-1}=0$ and by integration $\dot{x}^{2}+\lambda^{2} x^{2 \alpha}=1$ and thus $\dot{x}=\left(1-\lambda^{2} x^{2 \alpha}\right)^{1 / 2}$. Denoting by $y^{\prime}$ the derivative of $y$ with respect to $x$ we find

$$
y^{\prime}(x)=\frac{d y}{d t} \frac{d t}{d x}=-\frac{\lambda x^{2 \alpha}}{\left(1-\lambda^{2} x^{2 \alpha}\right)^{1 / 2}} .
$$

When $\alpha=1$ this differential equation coincides with the differential Equation (3.9). Then the boundary of the isoperimetric set

$$
E_{1}=\left\{(x, y) \in \mathbb{R}^{2}:|y|<\frac{1}{2}\left(\arccos |x|+|x| \sqrt{1-|x|^{2}}\right),|x|<1\right\}
$$

consists of two geodesics in the metric space $\left(\mathbb{R}^{2}, d_{1}\right)$ which are symmetric with respect to the $y$ axis.

This fact shows an interesting analogy with the Heisenberg group. Pansu conjectured in [19] that in this setting the solution to the isoperimetric problem is foliated by Heisenberg geodesics (see also [13] for some numerical evidence supporting this conjecture).

In $\mathbb{R}^{3}$ consider the vector fields $X=\partial_{x}+2 y \partial_{t}$ and $Y=\partial_{y}-2 x \partial_{t}$. The Heisenberg perimeter of a measurable set $E \subset \mathbb{R}^{3}$ is

$$
P_{H}(E)=\sup _{\varphi \in \mathcal{F}\left(\mathbb{R}^{2}\right)} \int_{E}\left(X \varphi_{1}(x, y, t)+Y \varphi_{2}(x, y, t)\right) d x d y d t,
$$

and the problem is to find a solution of

$$
\min \left\{P_{H}(E): E \subset \mathbb{R}^{3} \text { measurable set with }|E|=1\right\} \text {. }
$$

The existence of solutions is proved by Leonardi and Rigot in [14]. It is not known whether solutions are (up to a group left translation) smooth sets $E$ with rotational symmetry of the form $E=\{(z, t) \in \mathbb{C} \times \mathbb{R}:|t|<\psi(|z|)\}$. If this is the case, then the function $\psi$ can be computed and is $\psi(|z|)=\arccos |z|+|z| \sqrt{1-|z|^{2}}$ (see Proposition 3.4 in [16], Theorem 3.3 in [13], and [7]). This candidate solution is obtained (up to an immaterial constant factor $\frac{1}{2}$ ) by a rotation in the three-dimensional space of the set $E_{1}$ in (4.5) solving the isoperimetric problem in the Grushin plane for $\alpha=1$.

\section{Acknowledgments}

We would like to thank Matthieu Rickly for his comments on an early version of this article.

\section{References}

[1] Ambrosio, L. Fine properties of sets of finite perimeter in doubling metric measure spaces, Calculus of variations, nonsmooth analysis and related topics, Set-Valued Anal., 10(2-3), 111-128, (2002).

[2] Beckner, W. On the Grushin operator and hyperbolic symmetry, Proc. Am. Math. Soc., 129(4), 1233-1246, (2001).

[3] Bellaïche, A. The tangent space in sub-Riemannian geometry, in Sub-Riemannian Geometry, 1-78, Progr. Math., 144, Birkhäuser, Basel, (1996).

[4] Capogna, L., Danielli, D., and Garofalo, N. The geometric Sobolev embedding for vector fields and the isoperimetric inequality, Comm. Anal. Geom., 12, 203-215, (1994). 
[5] Chavel, I. Isoperimetric Inequalities, Differential Geometric and Analytic Perspectives, Cambridge Tracts in Mathematics, 145, Cambridge University Press, Cambridge, (2001).

[6] De Giorgi, E. Sulla proprietà isoperimetrica dell'ipersfera, nella classe degli insiemi aventi frontiera orientata di misura finita, Atti Accad. Naz. Lincei. Mem. Cl. Sci. Fis. Mat. Nat. Sez. I (8), 5, 33-44, (1958).

[7] Danielli, D., Garofalo, N., and Nhieu, D.M. Minimal surfaces, surfaces of constant mean curvature and isoperimetry in Carnot groups, preprint.

[8] Federer, H. and Fleming, W.H. Normal and integral currents, Ann. Math. (2), 72, 458-520, (1960).

[9] Franchi, B., Gallot, S., and Wheeden, R.L. Sobolev and isoperimetric inequalities for degenerate metrics, Math. Ann., 300, 557-571, (1994).

[10] Franchi, B. and Lanconelli, E. Une métrique associée à une classe d'opérateurs elliptiques dégénérés, Conference on linear partial and pseudodifferential operators, (Torino, 1982), Rend. Sem. Mat. Univ. Politec. Torino 1983. Special Issue, 105-114.

[11] Garofalo, N. and Nhieu, D.M. Isoperimetric and Sobolev inequalities for Carnot-Carathéodory spaces and the existence of minimal surfaces, Comm. Pure Appl. Math., 49, 1081-1144, (1996).

[12] Gromov, M. Carnot-Carathéodory spaces seen from within, Sub-Riemannian Geometry, 79-323, Progr. Math., 144, Birkhäuser, (1996).

[13] Leonardi, G. and Masnou, S. On the isoperimetric problem in the Heisenberg group, preprint (2002).

[14] Leonardi, G. and Rigot, S. Isoperimetric sets on Carnot groups, to appear in Huston J. Math.

[15] Maz'ya, V.G. Classes of domains and imbedding theorems for function spaces, Dokl. Acad. Nauk. SSSR, 133, $527-530$.

[16] Monti, R. Brunn-Minkowski and isoperimetric inequality in the Heisenberg group, to appear in Ann. Acad. Sci. Fenn. Math.

[17] Monti, R. and Cassano, F.S. Surface measures in Carnot-Carathéodory spaces, Calc. Var, 13, 339-376, (2001).

[18] Osserman, R. The isoperimetric inequality, Bull. Am. Math. Soc., 84(6), 1182-1238, (1978).

[19] Pansu, P. An isoperimetric inequality on the Heisenberg group, Conference on differential geometry on homogeneous spaces (Turin, 1983), Rend. Sem. Mat. Univ. Politec. Torino 1983, Special Issue, 159-174, (1984).

[20] Talenti, G. The Standard Isoperimetric Theorem, Handbook of Convex Geometry, vol. A, B, 73-123, North-Holland, Amsterdam, (1993).

[21] Varapoulos, N.Th., Saloff-Coste, L., and Coulhon, T. Analysis and Geometry on Groups, Cambridge Tracts in Mathematics, 100, Cambridge University Press, Cambridge, (1992).

Received May 13, 2003

Universität Bern, Mathematisches Institut, CH-3012 Sidlerstrasse 5, Bern, Schweiz e-mail: roberto.monti@math-stat.unibe.ch

Dipartimento di Matematica, Università di Bologna, Piazza di Porta San Donato, 5, 40127 Bologna, Italy e-mail: morbidel@dm.unibo.it 